\title{
An Analysis of Literature Reviews in the Context of Healthcare Program Assessment
}

\author{
Jalesa Martin ${ }^{1}$, Julia Leonard ${ }^{1}$, Shannon L. Sibbald ${ }^{1}$ \\ ${ }^{1}$ Faculty of Health Sciences, University of Western Ontario
}

\begin{abstract}
When conducting an assessment of existing literature, various types of literature reviews can be utilized. More specifically, each type has its advantages, disadvantages, and ideal circumstances in which it should be used. This paper explores the systematic review, scoping review, and rapid review in the context of research that aims to assess existing healthcare programs. Evidence suggests that the systematic review is the most rigorous and in-depth, but takes a significant amount of time to complete. The scoping review is less rigorous and used to identify what is known about a specific topic in the literature. The rapid review is similar in rigour to the systematic review, but takes less time and is often used in situations where data needs to be obtained quickly. In this paper, strengths, weaknesses, and examples of each review are given. They are then analyzed to see which review is the most suitable in the context of healthcare program assessment. In closing, it was decided that the rapid review is most suitable due to its limited time frame and extensive rigour, which is the most beneficial when conducting healthcare program assessment.
\end{abstract}

KEYWORDS: Systematic review, scoping review, rapid review, healthcare program assessment

\section{Introduction}

A literature review, defined as "an analysis of scientific literature materials about a specific topic", is one of the most highly requested articles (1). Although types of reviews primarily vary in structure and methodology, they provide valuable synthesized evidence when informing evidence-based practices (2). This paper aims to determine the most appropriate method to answer research questions that analyze evidence for the effectiveness of specific health programs. This paper will examine systematic, scoping, and rapid reviews. This will lead to the justification that the rapid review is the most appropriate method to answer this type of research question.

\section{Systematic Reviews}

Systematic reviews "seek to systematically search for, appraise, and synthesize research evidence, often adhering to the guidelines on the conduct of review provided by the Cochrane Collaboration" (2). They involve synthesizing the results of primary studies, which use strategies to limit the potential for bias and random error. Key steps include presenting a specific question, preparing a detailed research protocol, conducting a critical appraisal of primary studies, and statistically combining the retrieved data (3). This methodology enables focused research questions to be developed explicitly using four categories: "a specific population and setting, the condition of interest, an exposure to a test or treatment, and one or more specific outcomes" (3).

The effect of self-compassion on the selfregulation of health behaviors: $A$ systematic review is an example of a systematic review that was done in the field of health research. This article examines seven studies, highlighting the study design, intervention implementation, theoretical framework, and limitations of each component. The literature review was conducted using three search engines. Articles that were peer-reviewed, written in English, included selfregulation, and had measurable behaviour outcomes were used. For literature reviews that address gaps in the literature, it is recommended that researchers continuously conduct reviews and provide suggestions for future researchers who are examining a similar area of study (4). 
Systematic reviews aim to gather all known information on a topic, thus resulting in enhanced reliability. However, in recent years, different types of studies are being included that go beyond the sole inclusion of randomized control trials (2). This methodical approach has popularized systematic reviews in informing clinical policies and promoting the use of evidence-based practices (4).

One weakness of this method is that sometimes authors may refuse to include a study solely because of its design, which could neglect valuable information and limit the application of results. This may make it more difficult to answer complex research questions (2). The use of rigorous systematic reviews to summarize large amounts of evidence and highlight areas where research is needed provides valuable information for clinicians (4). However, conducting a systematic review requires a significant amount of time. The methodology used includes various steps of critical appraisal to maintain research quality and ensure a comprehensive literature search. For questions that analyze the evidence for effectiveness of health programs, quality and rigor are very important. However, the process and time required may go beyond the practicalities of the need for timely, evidenceinformed program assessment.

\section{Scoping Reviews}

Scoping reviews "provide a preliminary assessment of the potential size and scope of available research literature [with the] aim to identify the nature and extent of research evidence, usually including ongoing research" (2). When compared to a systematic review, this approach is less rigorous and requires less time. Moreover, scoping reviews can address gaps in evidence. In order to define the research question in a scoping review, there must be an understanding of the literature, gaps within the literature, and clarifications of definitions that are related to the question. As such, scoping reviews have become an increasingly efficient way to organize background information on a topic as they provide a broader picture of the evidence that can be used (5). Further, scoping reviews differ from systematic reviews as they identify parameters for the review and recognize the scope of systematic reviews, without describing detailed findings. Key steps of scoping reviews include determining a research question, identifying studies that are relevant, selecting a study, charting the available data, and collecting the results to provide a summary (5). This method can be used to examine the nature of the research activity, determine the cost of conducting a systematic review, summarize findings, or find gaps within the literature (6).

Patient engagement in Canada: a scoping review of the 'how' and 'what' of patient engagement in health research is an example of a scoping review that was done in the field of health research. This article aimed to identify various methods that influence outcomes of patient engagement. A review of 55 records demonstrated that there is no agreed upon standard for patient engagement in health research and no established framework to inform the process of patient engagement, limiting its uptake (7). The authors concluded that increasing the volume of evidence about patient engagement in the field of health research will help to normalize a patient's role beyond just being a participant and improve health outcomes in Canada (7).

Scoping reviews are very valuable to policy makers as they can indicate whether a full systematic review needs to be done. Notably, scoping reviews share many characteristics with systematic reviews, highlighting systematicity, transparency, and replicability (2). However, scoping reviews cannot be classified as the final product to a literature review as they do not have the rigor of a systematic review. Further, they often do not include a quality assessment of identified studies; leading to potential concern over the quality of studies used to determine conclusions. As a result, the findings often cannot be used to inform policy or practice (2).

Despite the reduced time span required for a scoping review and the ability to find gaps in literature, this method is not appropriate to answer this type of research question as it provides a broad picture without assessing quality or rigor. Also, it is often useful in disciplines with emerging evidence, while in established research areas, scoping reviews have been found to be ineffective (6). For example, a scoping review analyzing research questions about pediatric to adult care transition programs for patients with chronic illness, was not helpful, as this area has been studied for decades. In addition, the 
research questions used in scoping reviews often are quite broad, potentially leading to a lack of clarity and focus to proceed onto the next stages (6). In relation to the aforementioned research question, a crucial aspect is the quality of how programs are currently functioning, thus requiring detailed assessment; a scoping review would fail to address this quality.

\section{Rapid Reviews}

A rapid review is a "concession to the need for evidence-based decisions within a policymaker's time frame... and provides an assessment of what is already known about a policy or practice issue, by using systematic review methods to search and critically appraise existing research" (2). Rapid reviews are often completed in less than eight weeks and aim to synthesize results of primary studies through the use of error limiting strategies These include identifying specific exclusion and inclusion criteria and calculating the risk of bias assessments (8).

Self-management programs for Aboriginal and Torres Strait Islander Peoples with chronic conditions: A rapid review is an example of a rapid review that was done in the field of healthcare research. This article summarizes background knowledge on chronic conditions and how certain populations, such as the Aboriginal and Torres Strait Islander people, are at a higher risk of developing these conditions (9). Following the identification of inclusion and exclusion criteria, two search strategies were employed: a peer-reviewed search and a grey literature search - which includes literature that is not published or is available through channels other than journals. The results found limited peer-reviewed literature on the topic; however, sufficient evidence was found in grey literature that demonstrated characteristics of programs that would support successful selfmanagement in this population (9). The article concluded that, considering the complexity of this health issue, more evidence is needed to ensure that the information found in these studies is more applicable and shared within the context of peerreviewed literature (9).

One strength of rapid reviews is that: "they aim to be rigorous and explicit in method and thus systematic but make concessions to the breadth or depth of the process by limiting particular aspects of the systematic review process" (2). Conducting a rapid review involves the use of certain methods (i.e. broader search strategies) to shorten the length of time needed. This could include more inclusion and exclusion criteria, along with the use of interchangeable search terms and an increased variety of search engines. These techniques involve reviewing reviews, restricting the use of grey literature, and using only key variables (2).

A rapid review reduces the duration of the review process; however, this increases the risk of introducing bias, due to an emphasis on research of poorer quality and overlooking contradictions. More specifically, overlooking minimal or specific contradictions within the research. As a result of a compressed time frame, less attention to the question may result in a precise answer for the wrong question, or an answer that is inconclusive (2). The rapidity of a review does not dictate the quality. Nonetheless, concern arises when time saving measures alter the rigor and methodology. This can lead to process inadequacies, such as missing important evidence and an increased risk of analysis error in the results, while synthesizing evidence (8).

Despite this, conducting a rapid review serves to be the most suitable methodology to achieve research objectives in this context, whilst remaining within the scope of the project. Despite potential risk of error, a period of 8 weeks to conduct a rapid review provides the ability to promptly present summarized research evidence (8). Rapid reviews directly appeal to the healthcare field as they are able to support clinical and health policy decisions in a timely manner (8). As decision makers often do not focus specifically on the intricacies of research methods and the time-consuming nature of systematic reviews, rapid reviews are the best option in this case (8). Further, when compared to systematic reviews, which are considered the 'gold standard', rapid reviews maintain the same standards as systematic reviews (8). For example, a meta-epidemiological study done by Marshall et al. compared systematic reviews to rapid reviews and noted that the differences found between the reviews varied significantly (10). However, not all of the differences that were observed between the reviews were misleading - while in the case of timely healthcare program assessment, the high 
caliber of teams conducting rapid reviews, and smaller scope often leads to rates of prompt production (10). As such, rapid reviews should not be classified as inferior to systematic reviews as they produce similar outcomes (11).

This paper has examined the strengths and weaknesses of three prevalent types of literature reviews: systematic, scoping, and rapid reviews. The systematic review was compared to scoping reviews and rapid reviews to determine relative methodology effectiveness. Despite the use of systematic review characteristics to discredit the rapid review, a rapid review requires less time and rigor, yet upholds similar standards. Thus the criticisms of a rapid review compared to a systematic review are largely insubstantial in this context. In closing, a rapid review is the most suitable review methodology to answer questions that analyze evidence for the effectiveness of specific health programs. 


\section{References}

1. Garrard J. Health sciences literature review made easy: The matrix method. 3rd ed. Minneapolis: Jones \& Bartlett Learning; 2011.

2. Grant MJ, Booth A. A typology of reviews: an analysis of 14 review types and associated methodologies. Health Information \& Libraries Journal. 2009;26(2):91-108. DOI:10.1111/j.1471-1842.2009.00848.x

3. Stone PW. What is a Systematic Review? Applied Nursing Research. 2002;15(1):5253. DOI:10.1053/apnr.2002.29531

4. Biber DD, Ellis R. The effect of selfcompassion on the self-regulation of health behaviors: A systematic review. Journal of Health Psychology. 2017;24(14):2060-2071. DOI:10.1177/1359105317713361

5. Armstrong E, Hall BJ, Doyle J, Waters E. Cochrane Update 'Scoping the scope' of a cochrane review. Journal of Public Health. 2011;33(1):147-150.

DOI:10.1093/pubmed/fdr015

6. Levac D, Colquhoun H, Obrien KK. Scoping studies: advancing the methodology. Implementation Science. 2010;5(1). DOI:10.1186/1748-5908-5-69

7. Manafo E, Petermann L, Mason-Lai P, Vandall-Walker V. Patient engagement in Canada: a scoping review of the 'how' and 'what' of patient engagement in health research. Health Research Policy and Systems. 2018;16(1). 1-11.

DOI:10.1186/s12961-018-0282-4

8. Schünemann H, Moja L. Reviews: Rapid! Rapid! Rapid!...and systematic. Systematic Reviews. 2015;4(4): 1-3. DOI:10.1186/20464053-4-4.

9. Moore E, Lawn S, Oster C, Morello A. Selfmanagement programs for Aboriginal and Torres Strait Islander Peoples with chronic conditions: A rapid review. Chronic Illness. 2017;15(2): 83-123.

DOI:10.1177/1742395317750266
10. Marshall IJ, Marshall R, Wallace BC, Brassey J, Thomas J. Rapid reviews may produce different results to systematic reviews: a meta-epidemiological study. Journal of Clinical Epidemiology. 2019;109:30-41. DOI:10.1016/j.jclinepi.2018.12.015

11. Polisena J, Garritty C, Kamel C, Stevens A, Abou-Setta, AM. Rapid review programs to support health care and policy decision making: a descriptive analysis of processes and methods. Systematic Reviews. 2015;4(26):1-7. DOI:10.1186/s13643-0150022-6 